\title{
The secretion pattern of perivascular fat cells is different from that of subcutaneous and visceral fat cells
}

\author{
K. Rittig • J. H. Dolderer • B. Balletshofer • \\ J. Machann • F. Schick • T. Meile • M. Küper • \\ U. A. Stock $\cdot$ H. Staiger $\cdot$ F. Machicao $\cdot$ H.-E. Schaller • \\ A. Königsrainer $\cdot$ H.-U. Häring • D. I. Siegel-Axel
}

Received: 27 September 2011 / Accepted: 21 December 2011 / Published online: 13 February 2012

(C) Springer-Verlag 2012

\begin{abstract}
Aims/hypothesis We have previously found that the mass of perivascular adipose tissue (PVAT) correlates negatively with insulin sensitivity and post-ischaemic increase in blood flow. To understand how PVAT communicates with vascular vessels, interactions between perivascular, subcutaneous and visceral fat cells with endothelial cells (ECs) were examined with regard to inflammatory, metabolic and angiogenic proteins. To test for possible in vivo relevance of these findings, circulating levels of the predominant secretion product, hepatocyte growth factor (HGF), was measured in individuals carefully phenotyped for fat distribution patterns.
\end{abstract}

K. Rittig and J.H. Dolderer contributed equally to this study.

Electronic supplementary material The online version of this article (doi:10.1007/s00125-012-2481-9) contains peer-reviewed but unedited supplementary material, which is available to authorised users.

K. Rittig $\cdot$ B. Balletshofer $\cdot$ H. Staiger $\cdot$ F. Machicao $\cdot$

H.-U. Häring $•$ D. I. Siegel-Axel $(\bowtie)$

Department of Internal Medicine, Division of Endocrinology, Diabetology, Angiology, Nephrology and Clinical Chemistry,

Eberhard Karls University Tübingen,

Otfried-Müller Str. 10,

72076 Tübingen, Germany

e-mail: dorothea.axel@med.uni-tuebingen.de

J. H. Dolderer $\cdot$ H.-E. Schaller

Department of Plastic, Hand and Reconstructive Surgery,

BG-Trauma-Center, Eberhard Karls University,

Tübingen, Germany

J. Machann • F. Schick $\cdot$ H. Staiger · F. Machicao $•$ H.-U. Häring Institute for Diabetes Research and Metabolic Diseases of the Helmholtz Center Munich at the University of Tübingen

(Paul Langerhans Institute Tübingen),

Member of the German Center for Diabetes Research (DZD),

Tübingen, Germany
Methods Mono- and co-cultures of human primary fat cells with ECs were performed. mRNA expression and protein production were studied using Luminex, cytokine array, RealTime Ready and ELISA systems. Effects of HGF on vascular cells were determined by WST assays. In patients, HGF levels were measured by ELISA, and the mass of different fat compartments was determined by whole-body MRI.

Results In contrast with other fat cell types, PVAT cells released higher amounts of angiogenic factors, e.g. HGF, acidic fibroblast growth factor, thrombospondin-1, serpinE1, monocyte chemotactic protein-1 and insulin-like growth factor-binding protein-3. Cocultures showed different

J. Machann $\cdot$ F. Schick

Department of Experimental Radiology,

Eberhard Karls University,

Tübingen, Germany

T. Meile $\cdot$ M. Küper $\cdot$ A. Königsrainer

Department of General Visceral and Transplantation Surgery,

Eberhard Karls University,

Tübingen, Germany

U. A. Stock

Department of Thoracic, Cardiac and Vascular Surgery,

Eberhard Karls University,

Tübingen, Germany 
expression profiles from monocultures, and mature adipocytes differed from pre-adipocytes. HGF was preferentially released by PVAT cells and stimulated EC growth and smooth muscle cell cytokine release. Finally, in 95 patients, only PVAT, not visceral or subcutaneous mass, correlated independently with serum HGF levels ( $p=0.03 ; r=0.225$ ). Conclusions Perivascular (pre-)adipocytes differ substantially from other fat cells with regard to mRNA expression and protein production of angiogenic factors. This may contribute to fat tissue growth and atherosclerotic plaque complications. Higher levels of angiogenic factors, such as HGF, in patients with increased perivascular fat mass may have pathological relevance.

Keywords Adipocytes - Angiogenic factors · Atherosclerotic plaque $\cdot \mathrm{HGF} \cdot$ Insulin sensitivity . Perivascular tissue $\cdot$ Pre-adipocytes

\begin{tabular}{ll}
\multicolumn{2}{l}{ Abbreviations } \\
aFGF & Acidic FGF \\
bFGF & Basic FGF \\
EC & Endothelial cell \\
SMC & Smooth muscle cell \\
FGF & Fibroblast growth factor \\
G-CSF & Granulocyte colony-stimulating factor \\
HGF & Hepatocyte growth factor \\
IGFBP & Insulin-like growth factor-binding protein \\
MCP-1 & Monocyte chemotactic protein-1 \\
MMP-9 & Matrix metalloproteinase-9 \\
PVAT & Perivascular adipose tissue \\
SCAT & Subcutaneous adipose tissue \\
siRNA & Small interfering RNA \\
TIMP & Tissue inhibitor of metalloproteinase \\
TSP-1 & Thrombospondin-1 \\
VAT & Visceral adipose tissue \\
VEGF & Vascular endothelial growth factor
\end{tabular}

\section{Introduction}

Hypertension and insulin resistance of the skeletal muscle, liver and blood vessels are associated with obesity $[1,2]$. The latter association is dependent on the localisation and endocrine activity of the different fat tissues of the body. Mainly ectopic fat stores such as liver fat, but also visceral adipose tissue (VAT), show a significant association with whole-body insulin sensitivity [3]. However, adipose tissue is also an endocrine organ that secretes adipokines, which interact with the insulin signalling pathway $[4,5]$. Furthermore, inflammatory cytokines and angiogenic growth factors are released, influencing arterial endothelial function, smooth muscle cell (SMC) growth and atherosclerosis [6, 7]. Almost all arteries are encircled by notable amounts of perivascular adipose tissue (PVAT) [8]. It is present around all conduit arteries and in some microvascular beds, such as that of the mesentery [9]. There is evidence that the vascular adventitia, and predominantly perivascular fat, regulate endothelium-dependent vasodilatation and vascular tone, vessel wall thickness, angiogenesis and inflammation [10].

Previously, our group examined whether perivascular fat around the brachial artery influences NO signalling, reflected in flow-mediated dilation, or insulin sensitivity in humans. We found a close relationship between PVAT mass and insulin sensitivity [11]. Thus PVAT represents a novel and important fat compartment for the regulation of insulin sensitivity and vascular function independently of other fat compartments [12-17].

To understand how PVAT communicates with the vascular vessel wall and influences pathological processes, the study of interactions between adipocytes and endothelial cells (ECs) may provide important information. In embryogenesis, the development of adipose tissue is associated with vasculogenesis [18]. Interestingly, the appearance of adipose tissue occurs predominantly in well-vascularised regions [19]. Thus adipocyte-endothelial crosstalk is a main feature in adipose tissue and arterial vessel genesis. Anatomically, perivascular adipocytes are in close contact with arterial vessel wall cells and not separated by lamina $[8,20]$. There is even evidence that adipocytes infiltrate the outer regions of the adventitia [20]. Thus PVAT communicates with the arterial vessel wall by mediators secreted from adjacent adipocytes $[9,10]$. On the other hand, the adventitia of the vessel wall and perivascular tissue is interspersed with the vasa vasorum, which increases during vascular inflammation and injury [21]. In advanced plaques, a network of capillaries is formed by neovascularisation; they arise from the adventitial vasa vasorum and extend into the intimal layer of atherosclerotic lesions. They are proposed to be important regulators of plaque growth and lesion instability leading to acute coronary symptoms in patients [22]. It is already known that perivascular adipocytes contribute to the regulation of vascular tone and vascular remodelling [23]. However, limited data are available on interactions of PVAT with the arterial vessel wall in comparison with other fat tissues, especially with regard to the release of angiogenic factors [17, 24].

\section{Methods}

Cell isolation, differentiation and cultivation

Human pre-adipocytes were isolated from abdominal subcutaneous and visceral fat biopsy samples from five patients. In addition, perivascular fat biopsy samples were taken at the radial artery of seven patients. Arm arteries were 
used since the effects of our previous in vivo study were shown at the brachial artery [11]. The donors underwent abdominal, hand or coronary bypass surgery for clinical purposes; they gave informed written consent to the study. The age of the donors was between 56 and 67 years. The arteries used for EC isolation did not show any atherosclerotic changes.

The procedures were approved by the ethics committee of the University of Tübingen. Isolation, differentiation and cultivation of pre-adipocytes were performed as previously described $[25,26]$. The purity of primary cultures or potential contamination with ECs and monocytes/macrophages was determined by FACS analysis using specific antibodies (electronic supplementary material [ESM] Methods). Human arterial ECs were isolated from radial artery specimens, from heart patients, that had been discarded after bypass surgery, as described previously [27]. Potential contamination with SMCs or fibroblasts was tested by FACS analysis (ESM Methods) and double immunostaining using specific antibodies against von Willebrand factor and smooth muscle $\alpha$-actin, as described previously [27].

Transwell coculture system

Coculture experiments (ESM Methods) were performed in six-well dishes with polycarbonate membrane inserts (pore size 3.0, polycarbonate membrane, $24 \mathrm{~mm}$ insert; Corning, VWR International GmbH, Darmstadt, Germany).

Oil red staining and microscopy

Cellular lipid accumulation characteristic of mature, differentiated adipocytes was confirmed by oil red staining, as described previously [27]. Lipids stained red, and nuclei stained blue. The distribution and amounts of intracellular lipids were analysed with a light microscope.

\section{Cell growth assays}

ECs were seeded onto microtitre plates at a density of $5 \times 10^{3}$ cells $/ \mathrm{cm}^{2}$. After attachment, medium was renewed, and hepatocyte growth factor (HGF) $(1.0,5.0$ and $10 \mathrm{ng} / \mathrm{ml})$ or supernatant fraction collected from pre-adipocytes was added. In a second experiment, HGF small interfering RNA (siRNA) (a pool of three target-specific 19-25nucleotide siRNAs) or control siRNA (scrambled sequence) was added to pre-adipocytes to knockdown $H G F$ RNA expression $(H G F \alpha / \beta$ siRNA and control siRNA; Santa Cruz Biotechnology, Heidelberg, Germany). In addition, perivascular cell cultures were treated with an HGF-neutralising antibody (monoclonal mouse IgG1; R\&D Systems, Wiesbaden-Nordenstadt, Germany) in two different doses $(0.1$ and $0.5 \mu \mathrm{g} / \mathrm{ml})$. Finally, ECs were cultivated with the addition of these pretreated supernatant fractions. After
48 h, WST-1 tests (Roche Applied Science, Mannheim, Germany) were performed to measure the mitochondrial activity as an indicator of cell viability and cell proliferation.

\section{Multiplex analysis}

xMAP technology based on the Luminex platform (Luminex Corporation, Austin, TX, USA) was used. Three different panels were performed for the simultaneous detection of a great variety of angiogenic factors, cytokines and metabolic factors (ESM Methods).

Enzyme-linked immunosorbent assay

HGF concentrations were measured by sandwich enzyme immunoassays according to the manufacturer's recommendations (R\&D Systems). In addition, Human IL-6 ELISA MAX Deluxe and Human IL-8 ELISA MAX Deluxe (BioLegend Europe BV, Uithoorn, the Netherlands) were performed according to the manufacturer's instructions.

\section{Microarrays}

For the parallel determination of the relative levels of a large panel of human angiogenesis-related proteins, Proteome Profiler Human Angiogenesis Antibody Arrays (R\&D Systems) were performed (ESM Methods) according to the manufacturer's instructions.

\section{Real-time PCR analysis}

A real-time PCR analysis was used to quantify the transcripts of the angiogenic and inflammatory proteins of interest (ESM Methods). An mRNA screening experiment on a variety of adipogenic markers was also performed using the RealTime Ready system (Roche; ESM Methods).

Patient study

To study serum HGF release in humans, a total of 95 participants were studied (59 female, 36 male; median age 47 [19-66] years, Table 1). HGF was measured in serum samples by sandwich enzyme immunoassay. The examinations were performed in individuals from the Tübingen Lifestyle Intervention Program (TULIP) Study [28]. In brief, individuals at increased risk of developing type 2 diabetes were metabolically screened, and indicators of early atherosclerosis were determined. The individuals had one or more of the following diabetes risk factors: being overweight (BMI $>27 \mathrm{~kg} / \mathrm{m}^{2}$ ), being a first-degree relative of a patient with type 2 diabetes, or having impaired glucose tolerance. Details have been published previously [11]. Informed written consent was given by each participant. The 
Table 1 Demographics, anthropometrics and laboratory findings of the study population

\begin{tabular}{ll}
\hline Variable & All participants $(n=95)$ \\
\hline Age (years) & $47(19-66)$ \\
Sex (female/male) & $59 / 36$ \\
Systolic blood pressure $(\mathrm{mmHg})$ & $125(90-180)$ \\
Diastolic blood pressure $(\mathrm{mmHg})$ & $80(60-100)$ \\
BMI $\left(\mathrm{kg} / \mathrm{m}^{2}\right)$ & $27.7 \pm 4.9$ \\
Body fat $(\%)$ & $30.3 \pm 7.7$ \\
Visceral adipose tissue $(\%$ body weight) & $2.07(0.18-6.44)$ \\
Subcutaneous fat $(\%$ body weight $)$ & $22.67 \pm 9.0$ \\
Perivascular fat $\left(\mathrm{cm}^{2}\right)$ & $1.02(0.38-3.46)$ \\
\hline
\end{tabular}

Data are mean $\pm \mathrm{SD}$ when values are distributed normally, and median (range) if not normally distributed

study was approved by the ethics committee of the University of Tübingen, Germany.

\section{MRI examinations}

All MRI examinations were performed in the early morning after overnight fasting on a $1.5 \mathrm{~T}$ whole-body imager (Magnetom Sonata; Siemens Medical Solutions, Munich, Germany) (ESM Methods).

Presentation of data and statistics

The in vitro data are expressed as mean \pm SEM. Unpaired twotailed Student's $t$ test or one-way ANOVA (post hoc test: Bonferroni's multiple comparison test) was used to determine statistical significance. All statistical analyses were performed using Prism (GraphPad, La Jolla, CA, USA), considering a $p$ value of less than 0.05 as significant. Corresponding significance levels are indicated in the figures.

For statistical analysis of patient data, data are provided as mean $\pm \mathrm{SD}$ when values are distributed normally, and as median (range) when not normally distributed. Non-normally distributed variables were log-transformed to achieve normal distribution. Stepwise regression analysis was performed to check for determining factors of serum HGF levels. A $p$ value of less than 0.05 was considered to be significant. The analyses were conducted using the JMP statistical software package 4.0.4 (SAS Institute, Cary, NC, USA).

\section{Results}

Morphological features of perivascular adipocytes

Sufficient EC isolation was possible in $70 \%$ of vessel preparations. Immunostaining (ESM Fig. 1) and FACS analysis demonstrated the purity of the EC cultures used for the experiments. Sufficient pre-adipocytes could be isolated from each specimen of PVAT, VAT and subcutaneous adipose tissue (SCAT). FACS analysis (Fig. 1e-h) and
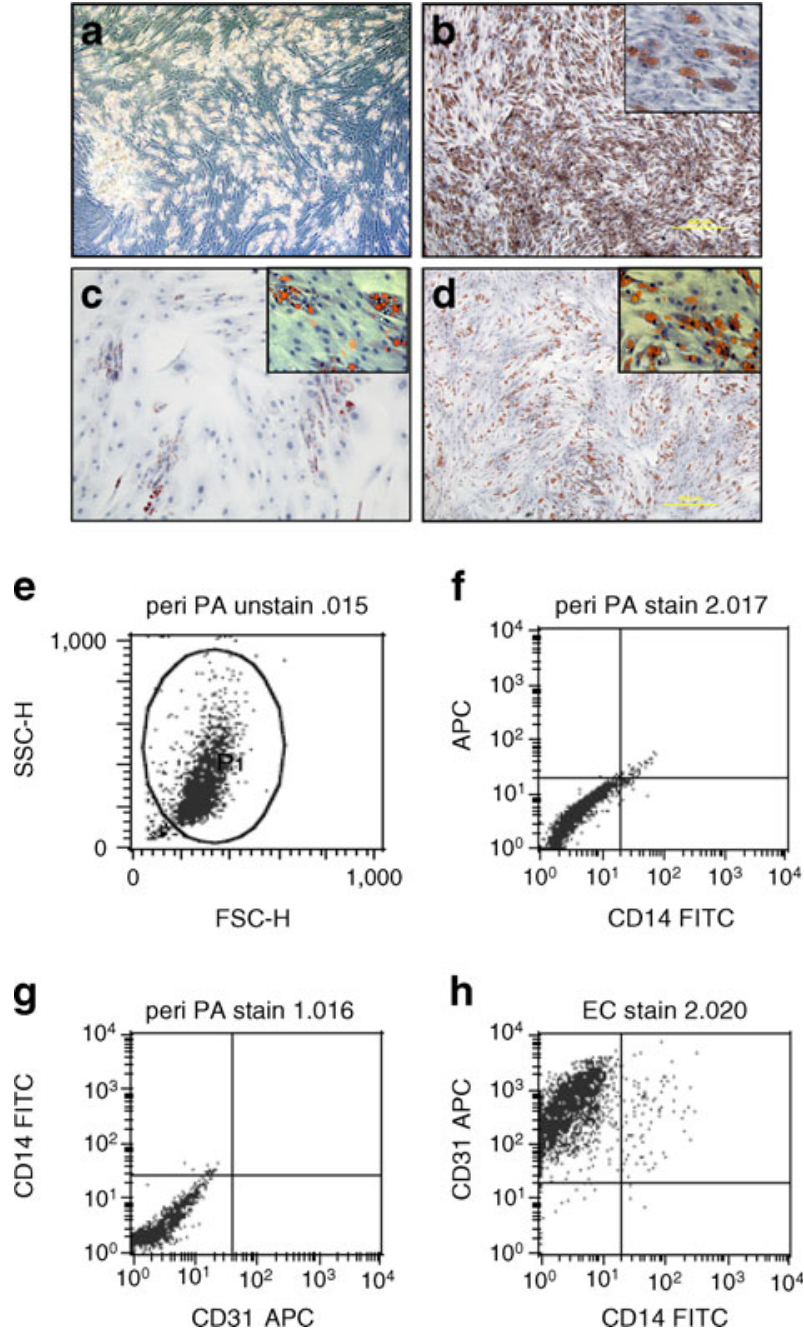

Fig. 1 Representative light microscopic presentation of human adipocytes after in vitro differentiation over 14 days, as described in the Methods section. Cells were fixed and imaged using phase-contrast microscopy (magnification $\times 4$ and as detail in the inset $\times 40$ ). a Unstained perivascular adipocytes show multiple intracellular lipid droplets and lipid cluster formation. b Oil red O-stained perivascular adipocytes with red-stained intracellular lipid droplet accumulation. c Oil red O-stained visceral adipocytes showing a more flat cell shape and only single islets of lipid-filled cell clusters. d Oil red O-stained subcutaneous adipocytes with the characteristic accumulation of redstained lipid droplets inside the cytoplasm. e-h FACS analysis at perivascular pre-adipocytes. e Image of the total perivascular preadipocyte (peri PA) population, side scatter height (SSC-H) versus forward scatter height (FCS-H). f Staining of the whole population with APC (allophycocyanin) and with specific antibodies against monocyte/macrophage marker CD14-FITC. $\mathbf{g}$ Double staining of perivascular preadipocytes (peri PA) with antibodies against the ECmarker CD31-APC and the monocyte/macrophage marker CD14FITC (fluorescein isothiocyanate). h Double staining of endothelial cells (EC) with antibodies against the monocyte/macrophage marker CD14-FITC and the EC-marker CD31-APC 
immunostaining showed that primary cultures from each cell isolation were not significantly contaminated with other cell types, except one culture containing some ECs, which was discarded. In culture, perivascular (Fig. 1a) and subcutaneous pre-adipocytes showed a spindle shape with cytoplasmic protrusions and cluster formation. When exposed to differentiation medium, both adipocyte types exhibited intense accumulation of cytoplasmic lipids. The cytoplasm of most cells was densely packed with lipids, as detected by oil red staining (Fig. 1b, d). In contrast, the cell shape of visceral adipocytes was more flat, and islets of lipid-filled cell clusters were observed by 14 days (Fig. 1c). Thus preadipocytes isolated from PVAT exhibited a comparable or even better capacity to differentiate into mature adipocytes than subcutaneous pre-adipocytes, but differed morphologically from visceral adipocytes. Pre-adipocyte cultures from seven independent patients showed that $>90 \%$ of cells differentiated into adipocytes. The degree of differentiation of subcutaneous pre-adipocytes was $>80 \%$. However, visceral pre-adipocytes were different because only about $30-60 \%$ differentiated.

Fat-depot-specific differences in protein release by pre-adipocytes and differentiated adipocytes

Cytokines and metabolic hormones With the use of multiplex analysis, protein release of up to 30 cytokines or metabolic hormones could be studied in parallel. In general, the secretion pattern of perivascular cells differed from visceral and subcutaneous cells. Perivascular preadipocytes secreted higher levels of monocyte chemotactic protein-1 (MCP-1) and vascular endothelial growth factor (VEGF) than visceral and subcutaneous pre-adipocytes (MCP-1: $275.9 \pm 64.9$ vs $106.5 \pm 48.6$ vs $54.8 \pm 22.2 \mathrm{pg} / \mathrm{ml}$, respectively, $p<0.05$; VEGF: $210.6 \pm 49.5$ vs $179.4 \pm 38.6$ vs $153.4 \pm 15.2 \mathrm{pg} / \mathrm{ml}$, respectively, NS). In contrast, visceral pre-adipocytes showed significantly higher levels of interleukins than perivascular and subcutaneous preadipocytes (IL-6: $393.1 \pm 61.5$ vs $227.7 \pm 41.2$ vs $60.1 \pm 6.6$ $\mathrm{pg} / \mathrm{ml}$, respectively, $p<0.05$; IL-8: $379.6 \pm 48.8$ vs $92.0 \pm 14.7$ vs $219.2 \pm 29.7 \mathrm{pg} / \mathrm{ml}$, respectively, $p<0.05$; IL- $12: 1.81 \pm 0.9$ vs $0.28 \pm 0.03$ vs $1.01 \pm 0.2 \mathrm{pg} / \mathrm{ml}$, respectively, $p<0.05$ ), confirming the more 'inflamed' character of this fat cell type.

Furthermore, pre-adipocytes differed from adipocytes. Significantly higher levels of basic fibroblast growth factor (bFGF) and MCP-1 were detected in supernatant fractions of all three fat cell types after differentiation. IL-6 levels were only higher in visceral adipocytes, leptin in perivascular adipocytes, and IL-1 receptor antagonist in both perivascular and subcutaneous adipocytes after differentiation. In contrast, in subcutaneous adipocytes, IL-6 and granulocyte colonystimulating factor (G-CSF) levels were lower after differentiation (Fig. 2).

Angiogenic factors Next, depot-dependent differences in the expression and release of angiogenic proteins were analysed. Indeed, all three pre-adipocyte tissue types showed different protein secretion profiles (Fig. 3). In supernatant fractions of perivascular pre-adipocytes, significantly higher levels of HGF, acidic FGF (aFGF), thrombospondin-1 (TSP-1), serpin-E1, MCP-1 and insulin-like growth factorbinding protein (IGFBP)-3 were detected compared with visceral pre-adipocytes (Fig. 3). In contrast, visceral preadipocytes secreted higher levels of IL-8. IGFBP-2 levels were lowest in subcutaneous preadipocytes compared with the others (Fig. 3c-j). Matrix metalloproteinase-9 (MMP-9) and endothelin-1 levels showed no differences between the different cell types.

To provide further support for the hypothesis that differentiation to mature adipocytes may also alter the release of angiogenic factors, pre-adipocytes were compared with adipocytes again. Among all tested proteins, secretion of HGF, IGFBP-1, IL-8, MMP-9 and TSP-1 was significantly lower
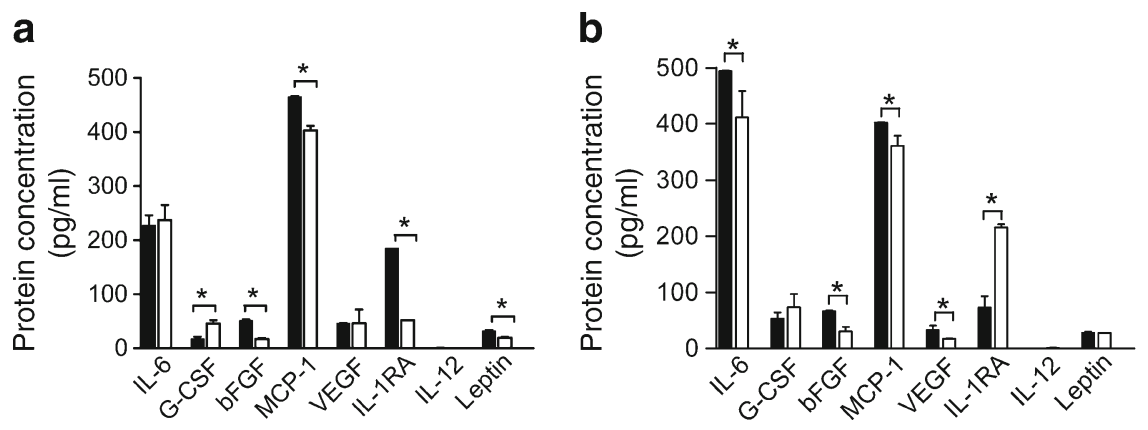

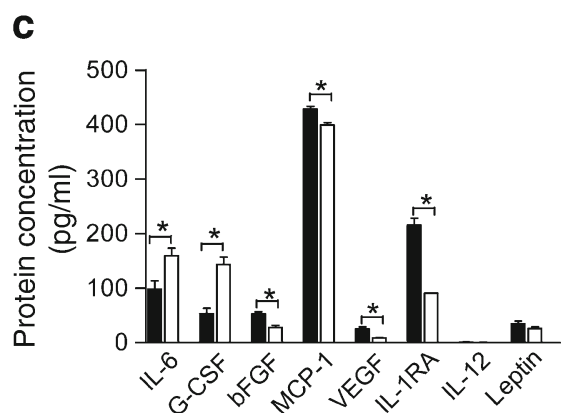

Fig. 2 Custom Human Cytokine Group I 8-plex (Luminex) for the determination of eight proteins in parallel. Comparison of protein release in supernatant fractions of pre-adipocytes (white bars) and differentiated adipocytes (black bars) after coculture of (a) perivascular preadipocytes, (b) visceral preadipocytes and (c) subcutaneous preadipocytes with ECs for $24 \mathrm{~h}$. Protein concentrations $(\mathrm{pg} / \mathrm{ml})$ are shown as mean \pm SEM in supernatant fractions of cells isolated from five (perivascular cells) or three (subcutaneous and visceral) different donors. IL-1RA, IL-1 receptor antagonist $\left({ }^{*} p<0.05\right.$ preadipocytes versus adipocytes) 
a

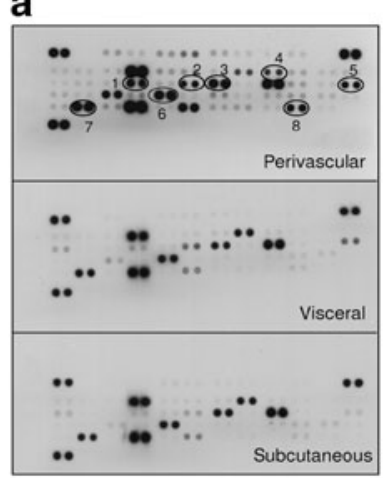

b

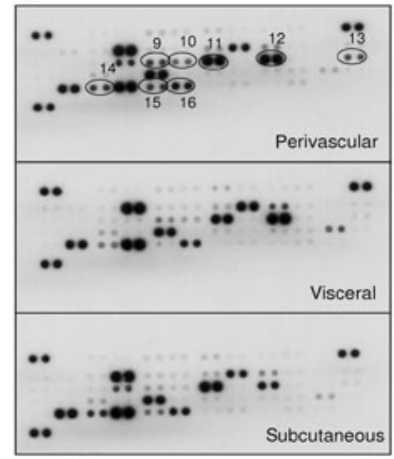

C

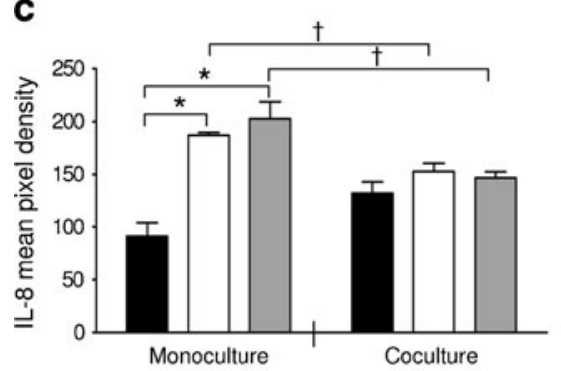

$\mathbf{f}$

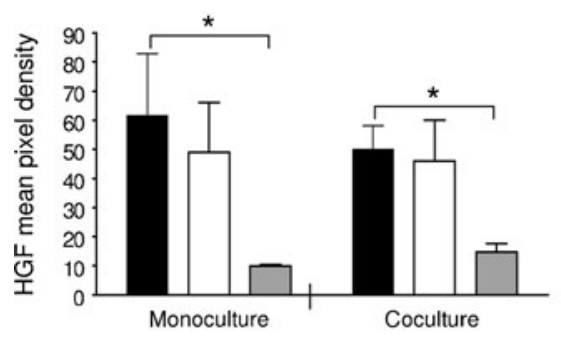

i

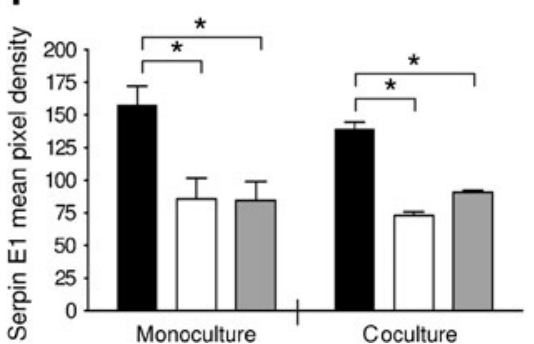

Fig. 3 a,b Representative proteome array membrane to detect up to 55 angiogenic candidates. Pre-adipocytes isolated from different fat depots were seeded on six-well plates at a density of $5 \times 10^{4}$ cells $/$ well and cultivated for 3 days. Primary human arterial endothelial cells were seeded on the upper side of gelatine-coated transwell inserts at a density of $5 \times 10^{4}$ cells/well and also cultivated for 3 days. After confluence had been reached, filter membranes covered with endothelial cells were inserted into the six-well plates containing confluent preadipocytes or adipocytes on the bottom of the culture dish. Both cell types were cultivated on their own as well as in cocultures for $24 \mathrm{~h}$. Then, conditioned medium from each compartment was collected separately and examined for protein production using the angiogenesis array membranes, as described in the Methods. Relevant amounts of a variety of angiogenic factors could be detected in conditioned medium
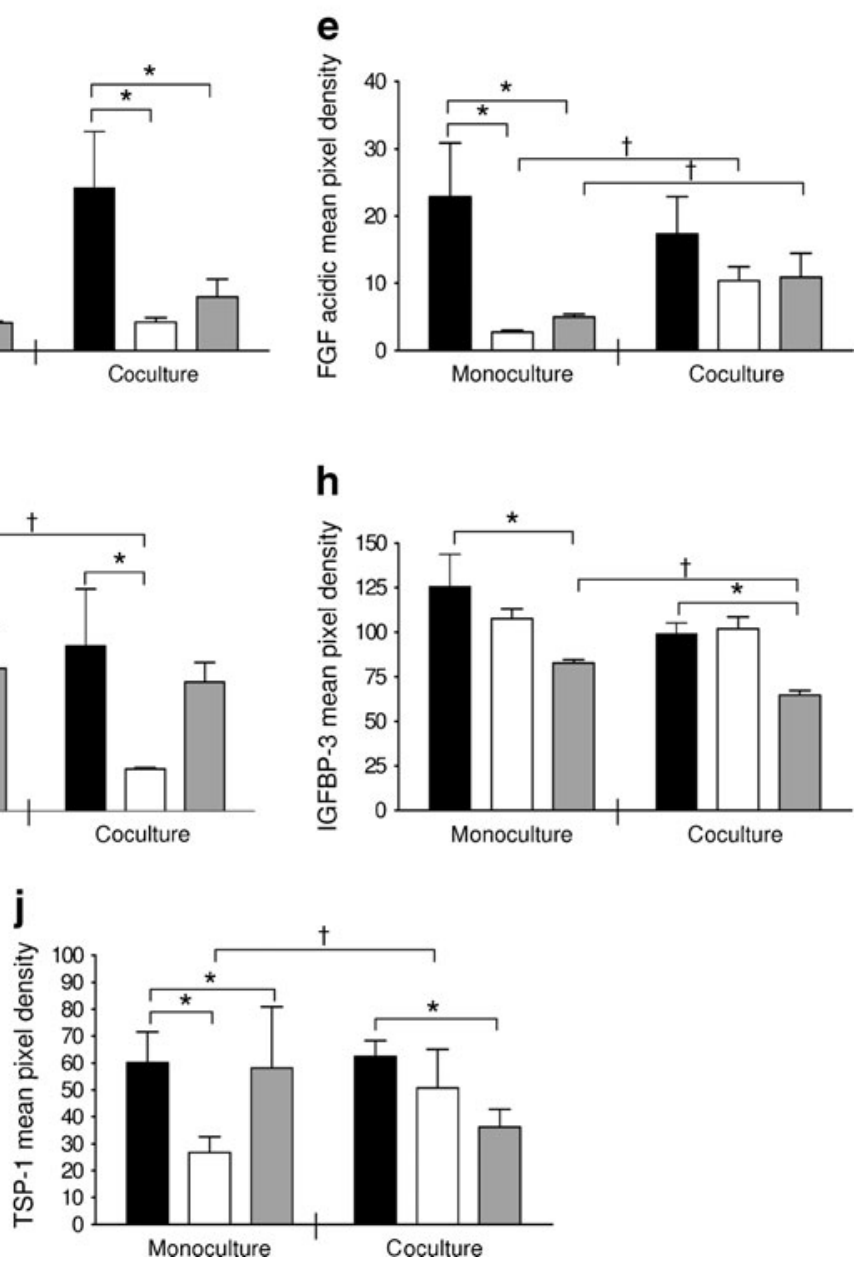

of perivascular, visceral and subcutaneous pre-adipocytes (a) and differentiated adipocytes (b). The angiogenic factors detected were: 1 , HGF; 2, IGFBP-2; 3, IGFBP-3; 4, aFGF; 5, MCP-1; 6, pentraxin-3; 7, serpin-E1; 8, VEGF; 9, IGFBP-1; 10, IGFBP-2; 11, IGFBP-3; 12, IL-8; 13, MCP-1; 14, serpin-F1; 15, TIMP-4; 16, TSP-1. c-j Quantification of protein production in supernatant fractions of fat cells: developed $\mathrm{x}$-ray films were quantified by scanning the film on a transmission-mode scanner, and absorbance measurements were obtained using the image analysis software. Perivascular pre-adipocytes (black bars) were compared with subcutaneous pre-adipocytes (white bars) and visceral preadipocytes (grey bars). Values are mean \pm SEM from three different donors. ${ }^{*} p<0.05$ for perivascular cells compared with visceral and subcutaneous pre-adipocytes and $\dagger p<0.05$ for monocultures versus cocultures 
after differentiation. In contrast, levels of serpin-E1, serpinF1 and pentraxin-3 increased after differentiation, whereas tissue inhibitor of metalloproteinase (TIMP)-1 was not changed (Fig. 4).

Since HGF is known to be a very potent angiogenic factor, differences in HGF secretion by all three cell types were studied in detail. HGF release was significantly higher in perivascular pre-adipocytes than in the others. Mature perivascular adipocytes also showed higher levels than visceral and subcutaneous adipocytes. However, in general, HGF concentrations were significantly lower in differentiated adipocytes than in pre-adipocytes (Fig. 5).

To underline the protein data, mRNA expression profiles were examined (Table 2). HGF mRNA expression was highest in both perivascular pre-adipocytes and mature adipocytes. In addition, $M C P-1$ (also known as CCL2), $I G F B P 3$ and $V E G F$ mRNA expression were higher in perivascular pre-adipocytes throughout the cultivation period of $72 \mathrm{~h}$ than in both of the other cell types. In addition, IL6 and $b F G F$ (also known as $F G F 2$ ) mRNA showed the highest expression in perivascular cells after only $1 \mathrm{~h}$ of cultivation, but decreased thereafter. In contrast, IL8 and TSPI mRNA expression was highest in visceral pre-adipocytes. The mRNA expression of these proteins was lower in subcutaneous pre-adipocytes. Thus the mRNA data confirm the protein array results and provide proof that perivascular cells express a significantly higher amount of angiogenic proteins.

In a further experimental setting, the mRNA expression of seven adipogenic marker proteins was analysed with the RealTime Ready system in both pre-adipocytes (Table 3) and differentiated adipocytes (ESM Table 1). The highest mRNA expression was found for serpin-1 and visfatin. In

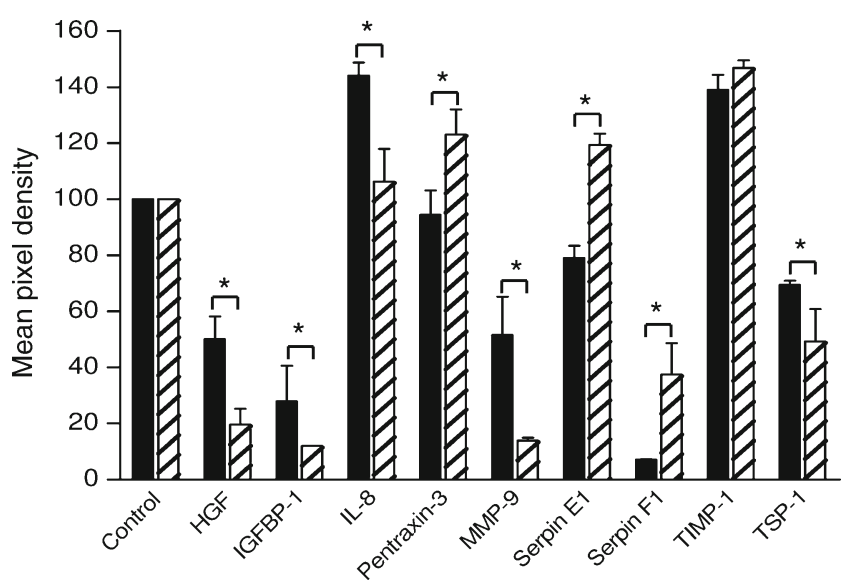

Fig. 4 Proteome Profiler Human Angiogenesis Array: densitometric calculation of protein levels. Perivascular pre-adipocytes (black bars) were compared with adipocytes (striped bars) treated with differentiation medium for 14 days. Values represent mean \pm SEM from three different donors. ${ }^{*} p<0.05$ for undifferentiated pre-adipocytes compared with mature adipocytes

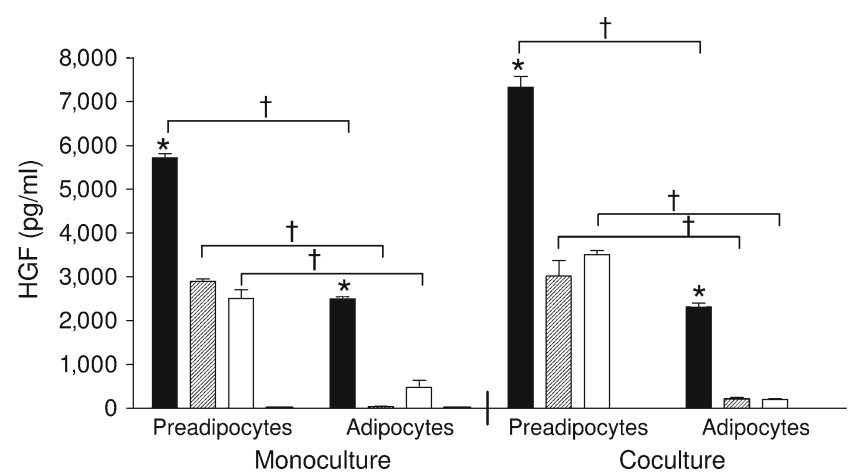

Fig. 5 Specific HGF ELISA to compare HGF secretion in mono- and co-cultures of all three fat cell types. Pre-adipocytes and differentiated adipocytes: HGF accumulation in supernatant fractions after $24 \mathrm{~h}$ was significantly higher in perivascular cultures (black bars) than in visceral (striped bars) and subcutaneous cells (white bars). Values represent mean \pm SEM of cells from three different donors. ${ }^{*} p<0.05$ for perivascular pre-adipocytes compared with visceral and subcutaneous preadipocytes; $\uparrow p<0.05$ for pre-adipocytes versus adipocytes

pre-adipocytes, adiponectin, leptin and resistin, and low expression of fetuin A and apelin, could also be detected in all three fat cell types, but without any significant difference. After differentiation to adipocytes, mRNA expression of all adipogenic proteins decreased markedly (ESM Table 1). Interestingly, ECs showed very high mRNA expression of apelin and visfatin.

\section{Influence of cocultured ECs on fat cells}

Protein array data in Fig. 3 indicate that the release of some proteins was altered after cocultivation with ECs, e.g. IL-8 and aFGF, whereas others remained unchanged, e.g. HGF and serpin-E1. Thus further analysis of the Luminex data was performed, which demonstrated that the levels of G-CSF and MCP-1 increased significantly in all three fat cell types under the influence of ECs, whereas IL-6 was only higher in subcutaneous cells. In contrast, VEGF levels decreased significantly. The concentrations of leptin and bFGF were not influenced at all (Fig. 6).

Then potential regulation of mRNA expression and protein production as a result of the time-dependent cocultivation was examined for some angiogenic proteins. HGF concentration in supernatant fractions of perivascular cells increased during cultivation, but without any differences between mono- and co-cultures. In contrast, HGF secretion by ECs increased significantly during cocultivation with perivascular cells up to $72 \mathrm{~h}$ (Fig. 7a). These data were confirmed at the mRNA level. $H G F$ mRNA was expressed constitutively in perivascular cells throughout the $72 \mathrm{~h}$ cocultivation period, but cocultivation induced significant upregulation of $H G F$ mRNA expression in ECs (Fig. 7b, c). Furthermore, $b F G F\left(\Delta / \Delta \mathrm{C}_{\mathrm{t}} 3.21 \pm 1.02\right.$ after $72 \mathrm{~h}$ vs $1.07 \pm$ 0.35 after $1 \mathrm{~h}, n=5, p<0.05), \operatorname{IL} 6\left(\Delta / \Delta \mathrm{C}_{\mathrm{t}} 9.38 \pm 6.07\right.$ vs 
Table 2 Fat-depot-dependent differences in expression profiles of angiogenic and proinflammatory proteins isolated from perivascular, visceral and subcutaneous pre-adipocytes after coculture with ECs

\begin{tabular}{|c|c|c|c|c|c|c|c|c|}
\hline Cells & $H G F$ & IL8 & $T S P 1$ & $I G F B P-3$ & $M C P-1$ & IL6 & $V E G F$ & $b F G F$ \\
\hline \multicolumn{9}{|l|}{$1 \mathrm{~h}$} \\
\hline $\mathrm{EC}$ & $0.9 \pm 0.3$ & $43 \pm 7$ & $1,104 \pm 192$ & $0.4 \pm 0.04$ & $9.6 \pm 5.6$ & $4.0 \pm 1.0$ & $3.4 \pm 0.4$ & $1.7 \pm 0.6$ \\
\hline Perivascular & $13 \pm 9$ & $11 \pm 9$ & $1,026 \pm 398$ & $129 \pm 58$ & $8.8 \pm 3.5$ & $49 \pm 15$ & $68 \pm 16$ & $48 \pm 14$ \\
\hline Visceral & $3.6 \pm 2.5$ & $30 \pm 29$ & $1,578 \pm 472$ & $40 \pm 2$ & $3.2 \pm 2.4$ & $31 \pm 12$ & $33 \pm 8$ & $48 \pm 12$ \\
\hline Subcutaneous & $1.2 \pm 0.8$ & $3.3 \pm 1.5$ & $1,008 \pm 132$ & $47 \pm 6$ & $0.7 \pm 0.4$ & $13 \pm 3$ & $35 \pm 14$ & $34 \pm 5$ \\
\hline \multicolumn{9}{|l|}{$72 \mathrm{~h}$} \\
\hline $\mathrm{EC}$ & $0.8 \pm 0.2$ & $32 \pm 13$ & $538 \pm 55$ & $0.2 \pm 0.04$ & $3.7 \pm 0.9$ & $1.1 \pm 0.7$ & $1.5 \pm 0.5$ & $0.7 \pm 0.1$ \\
\hline Perivascular & $24 \pm 4$ & $58 \pm 26$ & $376 \pm 186$ & $15 \pm 12$ & $7.8 \pm 6.1$ & $12 \pm 4$ & $10 \pm 2$ & $3.7 \pm 1.4$ \\
\hline Visceral & $12 \pm 0.4$ & $92 \pm 62$ & $473 \pm 5$ & $8.9 \pm 5.1$ & $2.7 \pm 0.9$ & $15 \pm 8$ & $8.9 \pm 4.1$ & $19 \pm 11$ \\
\hline Subcutaneous & $6.0 \pm 0.0$ & $65 \pm 5$ & $460 \pm 25$ & $8.0 \pm 1.0$ & $2.1 \pm 1.2$ & $4.7 \pm 2.2$ & $7.9 \pm 1.1$ & $7.1 \pm 1.5$ \\
\hline
\end{tabular}

mRNAs were determined by real-time PCR early (after $1 \mathrm{~h}$ ) or late (after $72 \mathrm{~h}$ ). Values $\left(\Delta \mathrm{C}_{\mathrm{t}}\right)$ are shown as mean \pm SEM of cells isolated from five (perivascular cells) or three (subcutaneous and visceral) different donors and represent gene expression of each protein relative to the RPS13 housekeeping gene product (ribosomal protein S13) as an endogenous reference

$1.30 \pm 0.35, n=5, p<0.05)$ and $I L 8\left(\Delta / \Delta \mathrm{C}_{\mathrm{t}} 15.11 \pm 10.80\right.$ vs $0.94 \pm 0.20, n=5, p<0.05)$ mRNA expression in preadipocytes was upregulated 3-18-fold as a result of the interaction with ECs. In the other fat cell types, mRNA expression of predominantly the proinflammatory and angiogenic cytokines, IL6 and $I L 8$, was upregulated after $72 \mathrm{~h}$ as a result of the cocultivation with ECs (ESM Table 2).

Angiogenic effects of HGF on human vascular ECs

To verify the effects of the potent angiogenic factor HGF, increasing doses of pure human HGF protein or supernatant fractions of perivascular pre-adipocytes were added to human arterial EC cultures. The WST assay showed that pure HGF stimulated EC growth dose-dependently with a maximum at a concentration of $10 \mathrm{ng} / \mathrm{ml}$ (Fig. 8a). This corresponds to the maximum concentrations $(5-15 \mathrm{ng} / \mathrm{ml})$ measured in conditioned medium of pre-adipocytes by
ELISA. Furthermore, growth stimulation induced by HGFcontaining supernatant fractions of perivascular cells was reduced significantly by preincubating perivascular cell cultures with the specific $H G F$ siRNA (Fig. 8b). In another independent approach, the stimulatory effect of supernatant fractions from perivascular cells was also reduced significantly and dose-dependently after neutralisation of HGF with an antibody (Fig. 8c).

Effects of perivascular HGF on atherosclerosis-related cells

We investigated further if HGF may influence vascular cells and consequently atherosclerosis. Protein release by human arterial smooth muscle was studied after $24 \mathrm{~h}$ of treatment with $10 \mathrm{ng} / \mathrm{ml}$ HGF. HGF induced the release of several cytokines, predominantly IL-6, but also IL-8, MCP-1 and the growth factor VEGF, compared with control cells without HGF. These array data were extended by a specific

Table 3 Fat-depot-dependent mRNA expression of seven adipogenic marker proteins

\begin{tabular}{lllll}
\hline Marker protein & Visceral & Subcutaneous & Perivascular & ECs \\
\hline Visaftin & $18.87 \pm 2.43$ & $19.62 \pm 2.76$ & $14.66 \pm 1.47$ & $40.53 \pm 5.66$ \\
Adiponectin & $0.0897 \pm 0.063$ & $0.0003 \pm 0.00003$ & $0.00002 \pm 0.000020$ & 0 \\
Leptin & $0.0007 \pm 0.0005$ & $0.028 \pm 0.018$ & $0.0029 \pm 0.0007$ & 0 \\
Resistin & $0.091 \pm 0.020$ & $0.379 \pm 0.039$ & $0.003 \pm 0.001$ & $0.105 \pm 0.07$ \\
Apelin & $0.018 \pm 0.003$ & $0.039 \pm 0.009$ & $0.053 \pm 0.018$ & $9.42 \pm 4.44$ \\
Fetuin A & $0.004 \pm 0.0004$ & $0.030 \pm 0.004$ & $0.003 \pm 0.002$ & $0.001 \pm 0.0009$ \\
Serpin-E1 & $795.81 \pm 64.55$ & $727.88 \pm 65.75$ & $527.43 \pm 91.51$ & $494.05 \pm 83.33$ \\
\hline
\end{tabular}

mRNAs were isolated from ECs and perivascular, visceral and subcutaneous pre-adipocytes. mRNA analysis was performed using the RealTime Ready System as described in ESM Methods. Values $\left(\Delta \mathrm{C}_{t}\right)$ represent gene expression of each protein relative to the RPS13 housekeeping gene product (ribosomal protein S13) as an endogenous reference 

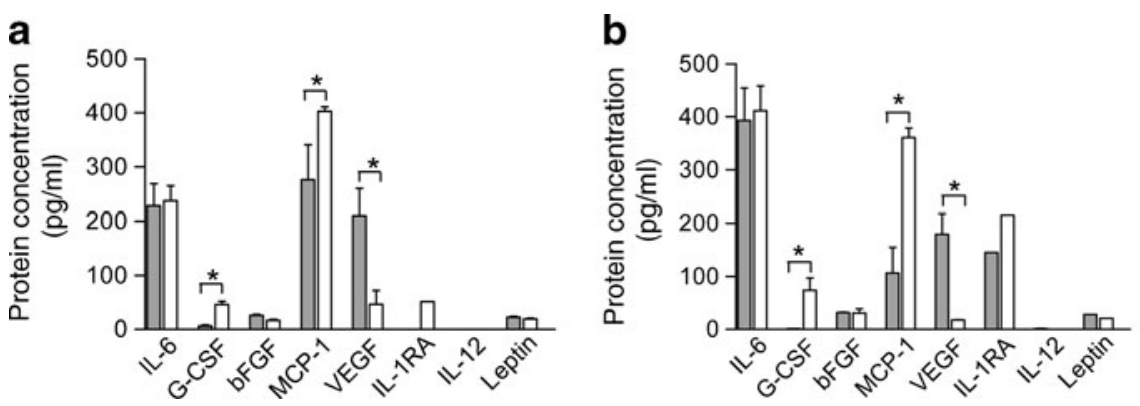

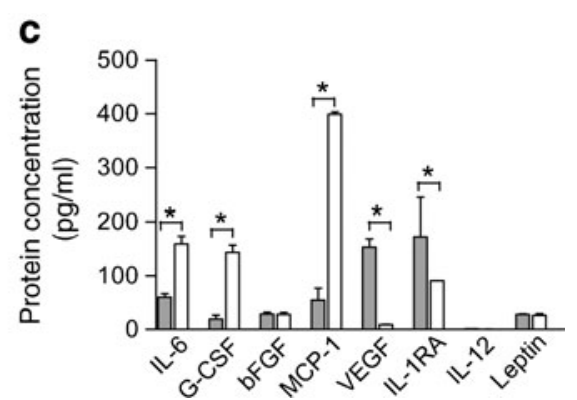

Fig. 6 Custom Human Cytokine Group I 8-plex (Luminex) for the determination of eight proteins in parallel. Comparison of protein release in monocultures (grey bars) compared with cocultures (white bars) of (a) perivascular preadipocytes, (b) visceral preadipocytes and (c) subcutaneous preadipocytes in the absence or presence of EC after
$24 \mathrm{~h}$. Protein concentrations $(\mathrm{pg} / \mathrm{ml})$ are shown as mean \pm SEM in supernatant fractions of cells isolated from five (perivascular cells) or three (subcutaneous and visceral) different donors $\left({ }^{*} p<0.05\right.$ monocultures versus cocultures)

Serum HGF levels and body fat distribution

ELISA, which showed comparable results. Significan stimulation of HGF-induced cytokine secretion was found for IL-6 $(37.16 \pm 6.27 \%, p<0.05)$ and IL-8 $(90.2 \pm 52.7 \%, p<0.05)$, which was an additional stimulation of protein release compared with unstimulated controls $(100 \pm 12.3 \%)$.
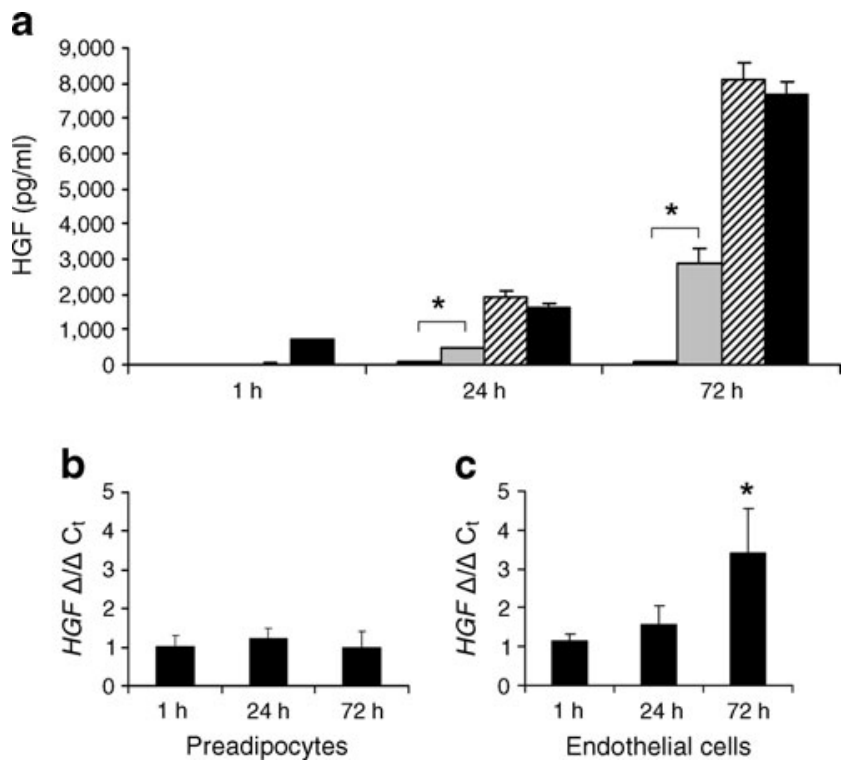

Fig. 7 a Quantification of HGF protein release by ECs (grey bars) and pre-adipocytes (black bars) in monocultures (striped bars) versus cocultures (filled bars). Cocultivation for $24 \mathrm{~h}$ and $72 \mathrm{~h}$ caused significant upregulation of HGF protein secretion in ECs $\left({ }^{*} p<0.05\right.$ monocultures versus cocultures) but not in pre-adipocytes. b $H G F$ mRNA expression determined by real-time PCR. In perivascular preadipocytes, $H G F$ mRNA was expressed constitutively throughout the $72 \mathrm{~h}$ cocultivation period without any differences between mono- and co-cultures. c In ECs, $H G F$ mRNA was upregulated by the cocultivation with perivascular pre-adipocytes and with increasing cocultivation time ( ${ }^{*} p<0.05$ mRNA expression after $72 \mathrm{~h}$ versus $\left.1 \mathrm{~h}\right)$. The relative gene expressions (cocultures versus monocultures) were calculated using cycle threshold $\left(\mathrm{C}_{\mathrm{t}}\right)$ values in accordance with the $\Delta / \Delta \mathrm{C}_{\mathrm{t}}$ method
We used MRI-based quantification of different fat compartments to determine the source of HGF in vivo. We have applied this method previously for other secretion products (e.g. RBP4) to judge the origin of the product [29]. Therefore HGF levels in 95 individuals were measured (Table 1), and the tissue mass of different fat compartments was determined by MRI.

In a multivariate model adjusted for age and sex, only PVAT mass correlated independently with serum HGF levels $(p=0.03 ; r=0.225)$, and VAT and SCAT did not show any significant association, although we observed a tendency for subcutaneous fat to do so $(p=0.07)$. In a second step, we performed a stepwise regression analysis with age, sex, perivascular, visceral and subcutaneous fat, and serum HGF levels as dependent variables. Here, only age and PVAT mass significantly influenced serum HGF levels (Table 4).

\section{Discussion}

The in vitro data of this study demonstrate that perivascular (pre-)adipocytes differ substantially from subcutaneous and visceral (pre-)adipocytes with regard to mRNA expression and protein production of inflammatory, metabolic and angiogenic proteins. To our knowledge, we are the first to show that perivascular fat cells in vitro and in vivo have a much higher capacity to secrete angiogenic factors, predominantly HGF, than other fat cell types. Furthermore, cocultures showed that arterial ECs influence the secretory behaviour of pre-adipocytes and mature adipocytes. Finally, our in vivo study suggests enhanced secretion of angiogenic factors, such as HGF, in patients with a greater perivascular fat mass, which may be of pathophysiological relevance.

Not only in fetal development but also in adults, adipose tissue grows and expands continuously, which is supported by an extensive capillary network [30]. It is well known that 
a

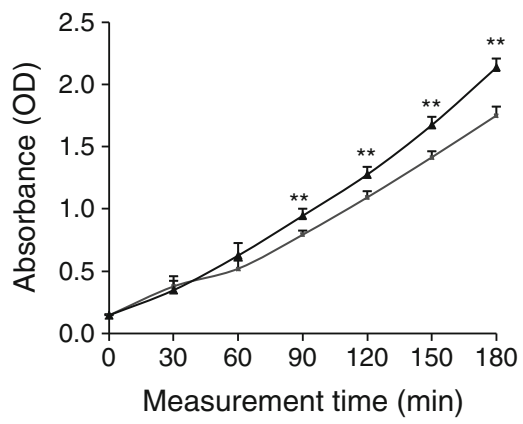

b

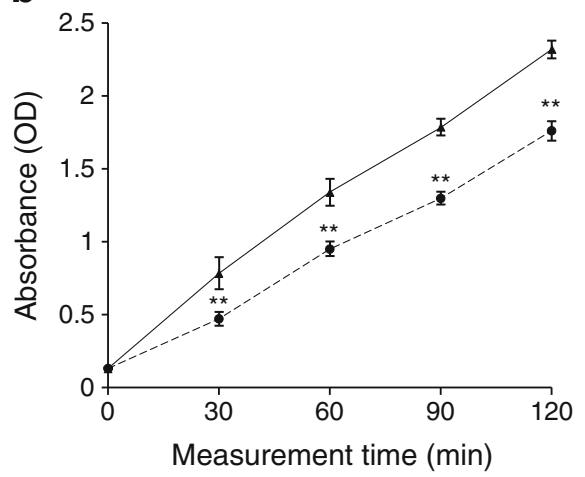

C

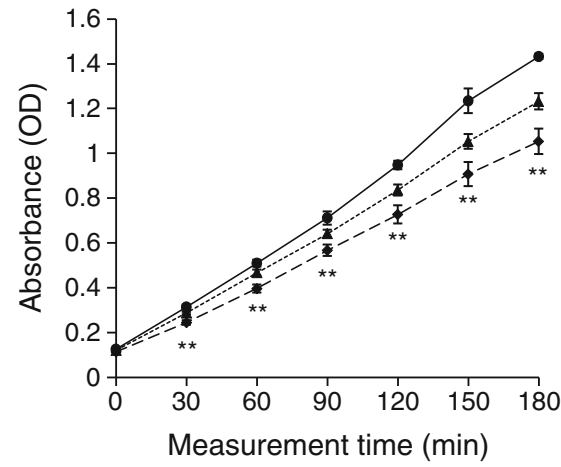

Fig. 8 WST assays: the absorbance reflects EC numbers/viability after $48 \mathrm{~h}$ of cultivation under the respective conditions. a $10 \mathrm{ng} / \mathrm{ml}$ pure HGF protein was added to human arterial EC cultures. HGF stimulated EC growth significantly. Values represent mean \pm SEM. $* * p<0.01$ for ECs treated with $10 \mathrm{ng} / \mathrm{ml}$ HGF (black triangles) compared with standard EC growth medium (black squares). b Perivascular cell cultures were treated with specific siRNA and control siRNA (scrambled sequence) or with (c) a neutralising antibody in two doses, and supernatant fractions were added to EC cultures. EC proliferation elicited by HGF which was secreted into supernatant fractions of perivascular cells (solid line) was reduced significantly $(* * p<0.01)$

arteries are larger and filled with lipid droplets. Furthermore, a study comparing adipokines expressed by periaortic and epicardial adipose tissue in post-mortem samples indicated that coronary PVAT seems to be more metabolically active than epicardial adipose tissue [38]. We showed that the amount and pattern of proteins secreted by perivascular fat cells differ from visceral and subcutaneous fat cells.

Furthermore, our coculture model enables the communication of fat cells with arterial vessel cells via the secretion of hormones, growth factors and cyokines. However, these cells are not simply mixed but separated by a porous membrane. Thus mRNA expression and protein production could be studied separately for each cell type after cocultivation. The interplay of perivascular cells with ECs in vivo may also cause altered release of growth factors, cytokines and adipokines, which may be critically related to EC and SMC dysfunction, vascular inflammation, angiogenesis and the development of atherosclerosis.

EC activation and angiogenesis may be important processes because intraplaque neovascularisation contributes to the progression of atherosclerosis and induces changes in SMC function [39]. Vulnerable atherosclerotic plaques prone to rupture show increased vasa vasorum and intraplaque haemorrhaging. We have provided proof that perivascular pre-adipocytes express significantly higher levels of angiogenic proteins than visceral and subcutaneous preadipocytes. Thus the location of perivascular fat cells near blood vessels and their higher angiogenic potential underscore the hypothesis that this specific fat depot influences the arterial vessel wall (patho)physiology and contributes to important features of atherosclerosis, such as plaque growth, vulnerability and differentiation.

HGF plays a special role because it is both a very potent angiogenic growth factor and a cytokine involved in

\begin{tabular}{lll}
\hline Variable & Estimate $(\beta)$ & $p$ value \\
\hline Age & 4.39 & 0.0004 \\
Sex & 0 & 0.8707 \\
Visceral adipose tissue & 0 & 0.9531 \\
Subcutaneous adipose tissue & 0 & 0.4464 \\
Perivascular adipose tissue & 75.87 & 0.0240 \\
\hline
\end{tabular}


haematopoiesis and vasculogenesis [40-42]. HGF is in part attributable to excess adipose tissue, and circulating levels of HGF are raised in obese patients, and this correlates linearly with BMI [43]. Finally, HGF can directly contribute to the progression of atherosclerosis by plaque neovascularisation $[44,45]$. However, the main source of raised serum HGF levels in obese patients is not known. Our study provides evidence that perivascular fat cells express significantly higher levels of HGF than subcutaneous and visceral fat cells. HGF stimulated EC growth dose-dependently. These results and the blocking experiments imply that HGF may, at least in part, be responsible for the growth-stimulatory effects of proteins secreted by perivascular fat cells. Interestingly, functional changes in SMCs were also induced. Secretion of the proinflammatory proteins, IL-6 and IL-8, was significantly stimulated after treatment of SMCs with HGF. Vascular SMC phenotypic modulation plays a key role in atherosclerosis and is characterised by a switch from a 'contractile' phenotype to a 'synthetic' phenotype. SMCs change into a 'proinflammatory' phenotype, secreting interleukins, which stimulate monocyte and macrophage adhesion and other processes during atherosclerosis [46]. Thus we have provided some evidence that perivascular fat cells alter the growth and function of vascular cells and may be involved in processes supporting atherosclerosis.

To prove whether our basic findings also correlate with an angiogenic secretion profile of perivascular fat in vivo, we tested whether HGF is mainly expressed in PVAT cells of humans and released into the serum. We studied serum HGF levels in patients with low and high amounts of PVAT, VAT and SCAT. Ninety-five individuals underwent whole-body MRI to measure fat distribution. In our univariate analysis, PVAT was the only fat compartment that correlated with serum HGF levels. In a stepwise regression analysis including PVAT, VAT, SCAT, age and sex, only age and PVAT were found to be determining factors for serum HGF levels. This finding underlines the notion that PVAT may be the main fat compartment for HGF expression in humans.

This study extends our earlier work showing that the mass of PVAT correlated significantly with insulin sensitivity and the post-ischaemic increase in blood flow, and also with inflammation as reflected by high sensitive CRP [11]. Thus the mass of PVAT is not merely an epiphenomenon of adiposity. Furthermore, determination of adipose tissue around the thoracic aorta in the Framingham Heart Study population showed a significant correlation of this fat compartment with BMI, visceral obesity, hypertension and diabetes. In addition, perivascular fat was found to be associated with coronary calcifications [47]. Finally, in a segment analysis study, the amount of pericoronary fat was associated with plaque burden characteristic of advanced coronary atherosclerosis [14]. Together, these studies indicate clearly that the amount and function of perivascular fat is closely associated with the most prominent risk factors leading to cardiovascular diseases.

In conclusion, our data provide new insights into the special nature of perivascular fat cells. Both perivascular pre-adipocytes and mature adipocytes exhibit a different expression profile and amount of angiogenic, growth stimulatory, inflammatory and metabolic factors compared with cells from other fat stores. PVAT represents a specialised, functionally very active fat tissue, which may contribute in excess, e.g. in patients with diabetes and obesity, to arterial vessel wall diseases. Further clinical studies may underline the importance of perivascular tissue in obesity, diabetes and cardiovascular complications.

Acknowledgements We would like to thank U. Schmidt and B. Schreiner for excellent technical assistance.

Funding This study was funded by a grant from the German Federal Ministry for Education and Research (BMBF), joint research project 'Gesundheitsregion REGINA', promotional reference 01KQ0902F, and in part by a grant from the BMBF to the German Center for Diabetes Research (DZD e.V.).

Duality of interest The authors declare that there is no duality of interest associated with this manuscript.

Contribution statement All authors contributed to conception and design, or analysis and interpretation of data, to drafting the article or revising it critically for important intellectual content, and to final approval of the version to be published. KR, JHD, HUH and DISA were mainly responsible for the conception of the study, drafting the article, and the intellectual input, while HS and FM and contributed to the redrafting and revision of the manuscript. BB, JM and FS contributed to the conception, design and data collection of the clinical study. TM, MK, UAS, HES and AK provided specimens from patients.

\section{References}

1. Gallagher EJ, Leroith D, Karnieli E (2010) Insulin resistance in obesity as the underlying cause for the metabolic syndrome. Mt Sinai J Med 77:511-523

2. Iozzo P (2009) Viewpoints on the way to the consensus session: where does insulin resistance start? The adipose tissue. Diabetes Care 32(Suppl 2):S168-S173

3. Despres JP, Lemieux I (2006) Abdominal obesity and metabolic syndrome. Nature 444:881-887

4. Wozniak SE, Gee LL, Wachtel MS, Frezza EE (2009) Adipose tissue: the new endocrine organ? A review article. Dig Dis Sci 54:1847-1856

5. Hauner H (2005) Secretory factors from human adipose tissue and their functional role. Proc Nutr Soc 64:163-169

6. Ohman MK, Shen Y, Obimba CI et al (2008) Visceral adipose tissue inflammation accelerates atherosclerosis in apolipoprotein E-deficient mice. Circulation 117:798-805

7. Mazurek T, Zhang L, Zalewski A et al (2003) Human epicardial adipose tissue is a source of inflammatory mediators. Circulation 108:2460-2466 
8. Ouwens DM, Sell H, Greulich S, Eckel J (2010) The role of epicardial and perivascular adipose tissue in the pathophysiology of cardiovascular disease. J Cell Mol Med 14:223-2234

9. Guzik TJ, Marvar PJ, Czesnikiewicz-Guzik M, Korbut R (2007) Perivascular adipose tissue as a messenger of the brain-vessel axis: role in vascular inflammation and dysfunction. J Physiol Pharmacol 58:591-610

10. Rajsheker S, Manka D, Blomkalns AL, Chatterjee TK, Stoll LL, Weintraub NL (2010) Crosstalk between perivascular adipose tissue and blood vessels. Curr Opin Pharmacol 10:191-196

11. Rittig K, Staib K, Machann J et al (2008) Perivascular fatty tissue at the brachial artery is linked to insulin resistance but not to local endothelial dysfunction. Diabetologia 51:2093-2099

12. Yudkin JS, Eringa E, Stehouwer CD (2005) "Vasocrine" signalling from perivascular fat: a mechanism linking insulin resistance to vascular disease. Lancet 365:1817-1820

13. Ichiki T (2010) Perivascular adipose tissue, a Janus-faced regulator of vascular function. Circ J 74:1300-1301

14. Mahabadi AA, Reinsch N, Lehmann N et al (2010) Association of pericoronary fat volume with atherosclerotic plaque burden in the underlying coronary artery: a segment analysis. Atherosclerosis 211:195-199

15. Silaghi AC, Poantă L, Valea A, Pais R, Silaghi H (2011) Is epicardial adipose tissue, assessed by echocardiography, a reliable method for visceral adipose tissue prediction? Med Ultrason 13:15-20

16. Sicari R, Sironi AM, Petz R et al (2011) Pericardial rather than epicardial fat is a cardiometabolic risk marker: an MRI vs echo study. J Am Soc Echocardiogr 24:1156-1162

17. Silaghi A, Piercecchi-Marti MD, Grino M et al (2008) Epicardial adipose tissue extent: relationship with age, body fat distribution, and coronaropathy. Obesity 16:2424-2430

18. Hausman GJ, Richardson RL (2004) Adipose tissue angiogenesis. J Anim Sci 82:925-934

19. Crandall DL, Hausman GJ, Kral JG (1997) A review of the microcirculation of adipose tissue: anatomic, metabolic, and angiogenic perspectives. Microcirculation 4:211-232

20. Chatterjee TK, Stoll LL, Denning GM et al (2009) Proinflammatory phenotype of perivascular adipocytes: influence of high-fat feeding. Circ Res 104:541-549

21. Kwon HM, Sangiorgi G, Ritman EL et al (1998) Enhanced coronary vasa vasorum neovascularization in experimental hypercholesterolemia. J Clin Invest 101:1551-1556

22. Mulligan-Kehoe MJ (2010) The vasa vasorum in diseased and nondiseased arteries. Am J Physiol Heart Circ Physiol 298: H295-H305

23. Engeli S (2005) Is there a pathophysiological role for perivascular adipocytes? Am J Physiol Heart Circ Physiol 289:H1794-H1795

24. Henrichot E, Juge-Aubry CE, Pernin A et al (2005) Production of chemokines by perivascular adipose tissue: a role in the pathogenesis of atherosclerosis? Arterioscler Thromb Vasc Biol 25:2594-2599

25. Schling P, Mallow H, Trindl A, Loffler G (1999) Evidence for a local renin angiotensin system in primary cultured human preadipocytes. Int J Obes Relat Metab Disord 23:336-341

26. Van RL, Bayliss CE, Roncari DA (1976) Cytological and enzymological characterization of adult human adipocyte precursors in culture. J Clin Invest 58:699-704

27. Axel DI, Brehm BR, Wolburg-Buchholz K, Betz EL, Köveker G, Karsch KR (1996) Induction of cell-rich and lipid-rich plaques in a transfilter coculture system with human vascular cells. J Vasc Res 33:327-339

28. Schafer S, Kantartzis K, Machann J et al (2007) Lifestyle intervention in individuals with normal versus impaired glucose tolerance. Eur J Clin Invest 37:535-543
29. Stefan N, Hennige AM, Staiger H et al (2007) High circulating retinol-binding protein 4 is associated with elevated liver fat but not with total, subcutaneous, visceral, or intramyocellular fat in humans. Diabetes Care 30:1173-1178

30. Rupnick MA, Panigrahy D, Zhang CY, Dallabrida SM, Lowell BB, Langer R, Folkman MJ (2002) Adipose tissue mass can be regulated through the vasculature. Proc Natl Acad Sci U S A 99:10730-10735

31. Lijnen HR (2008) Angiogenesis and obesity. Cardiovasc Res 1 (78):286-293

32. Cao Y (2007) Angiogenesis modulates adipogenesis and obesity. J Clin Invest 117:362-2368

33. Lai N, Jayaraman A, Lee K (2009) Enhanced proliferation of human umbilical vein endothelial cells and differentiation of 3T3-L1 adipocytes in coculture. Tissue Eng Part A 15:10531061

34. Saiki A, Watanabe F, Murano T, Miyashita Y, Shirai K (2006) Hepatocyte growth factor secreted by cultured adipocytes promotes tube formation of vascular endothelial cells in vitro. Int $\mathrm{J}$ Obes (Lond) 30:1676-1684

35. Aoki S, Toda S, Sakemi T, Sugihara H (2003) Coculture of endothelial cells and mature adipocytes actively promotes immature preadipocyte development in vitro. Cell Struct Funct 28:5560

36. Poissonnet CM, Burdi AR, Bookstein FL (1983) Growth and development of human adipose tissue during early gestation. Early Hum Dev 8:1-11

37. Frye CA, Wu X, Patrick CW (2005) Microvascular endothelial cells sustain preadipocyte viability under hypoxic conditions. In Vitro Cell Dev Biol Anim 41:160-164

38. Spiroglou SG, Kostopoulos CG, Varakis JN, Papadaki HH (2010) Adipokines in periaortic and epicardial adipose tissue: differential expression and relation to atherosclerosis. $\mathrm{J}$ Atheroscler Thromb $17: 115-130$

39. Di Stefano R, Felice F, Balbarini A (2009) Angiogenesis as risk factor for plaque vulnerability. Curr Pharm Des 15:1095-1106

40. Nakagami H, Kaneda Y, Ogihara T, Morishita R (2005) Hepatocyte growth factor as potential cardiovascular therapy. Expert Rev Cardiovase Ther 3:513-519

41. Funakoshi H, Nakamura T (2003) Hepatocyte growth factor: from diagnosis to clinical applications. Clin Chim Acta 327:1-23

42. Bell LN, Cai L, Johnstone BH, Traktuev DO, March KL, Considine RV (2008) A central role for hepatocyte growth factor in adipose tissue angiogenesis. Am J Physiol Endocrinol Metab 294: E336-E344

43. Rehman J, Considine RV, Bovenkerk JE et al (2003) Obesity is associated with increased levels of circulating hepatocyte growth factor. J Am Coll Cardiol 41:1408-1413

44. Chowdhury M, Ghosh J, Slevin M, Smyth JV, Alexander MY, Serracino-Inglott F (2010) A comparative study of carotid atherosclerotic plaque microvessel density and angiogenic growth factor expression in symptomatic versus asymptomatic patients. Eur J Vasc Endovasc Surg 39:388-395

45. Liu Y, Wilkinson FL, Kirton JP et al (2007) Hepatocyte growth factor and c-Met expression in pericytes: implications for atherosclerotic plaque development. J Pathol 212:12-19

46. Orr AW, Hastings NE, Blackman BR, Wamhoff BR (2010) Complex regulation and function of the inflammatory smooth muscle cell phenotype in atherosclerosis. J Vasc Res 47:168-180

47. Lehman SJ, Massaro JM, Schlett CL, O'Donnell CJ, Hoffmann U, Fox CS (2010) Peri-aortic fat, cardiovascular disease risk factors, and aortic calcification: the Framingham Heart Study. Atherosclerosis 210:656-661 